\title{
Stage of readiness for planned activity reveals heart failure patients at higher risk
}

\author{
Tracie R. Parish ${ }^{1^{*}}$, Maria Kosma ${ }^{2}$, Michael A. Welsch ${ }^{2}$ \\ ${ }^{1}$ Department of Kinesiology and Health Studies, Southeastern Louisiana University, Hammond, USA; \\ *Corresponding Author: tracie.parish@selu.edu \\ ${ }^{2}$ Department of Kinesiology, Louisiana State University, Baton Rouge, USA
}

Received 5 November 2012; revised 6 December 2012; accepted 14 December 2012

\section{ABSTRACT}

Few individuals with chronic heart failure (CHF) engage in regular physical activity. PURPOSE: 1) To examine stage of readiness for planned physical activity; 2) To compare estimated selfreported daily physical activity and exercise tolerance/capacity by stage of readiness; and 3) To determine the association between stages of readiness for planned physical activity, selfreported daily physical activity and exercise tolerance, in CHF patients. METHODS: Onehundred eleven CHF patients (Age: $53 \pm 14$ yrs; New York Heart Association class II/III) participated. Each participant's stage of readiness for planned physical activity was assessed. Patients completed a self-reported daily physical activity questionnaire, and performed a sixminute walk test. RESULTS: Average left ventricular ejection fraction (LVEF) was $30.12 \% \pm$ $10.72 \%$. Twelve patients were in Precontemplation (PC), 29 in Contemplation (C), 30 in Preparation (PR), 20 in Action (A), and 20 in Maintenance $(M)$. There were no differences in age and LVEF between stages. Those classified in A/M performed more minutes in activities $>3$ METs. Average 6MWD was $349 \pm 118$ meters, with significant differences between stages (PC, C $<$ PR $<A<M ; p<0.01$ ). Thirty-four of 71 patients in preaction scored $<300$ meters versus only 3 and 5 of $\mathbf{4 0}$ from A/M. CONCLUSION: The majority of CHF patients are in pre-action stages of readiness for adoption of planned physical activity. Patients in pre-action are engaged in less daily activity and have lower exercise tolerance/capacity than those in A/M. Lower exercise tolerance/capacity suggests these patients are more fragile and at greater risk for complications and early mortality. Greater emphasis should be placed on strategies to move patients toward A/M.
Keywords: Chronic Heart Failure; Transtheoretical Model; Daily Activity; Exercise Tolerance

\section{INTRODUCTION}

Chronic heart failure (CHF) remains a significant health problem in the United States, with frequent hospitalizations, widespread functional impairment, and a high mortality rate. Recent studies have shown the significant value of the use of a multidisciplinary approach in the management of the heart failure patient. These programs emphasize the importance of close monitoring and patient education $[1,2]$. Despite positive outcomes from these programs, including fewer readmissions and hospital days, decreased medical costs, and improved functional status and quality of life, patient participation and adherence remain suboptimal $[2,3]$.

A major component of the management of the CHF patient is planned physical activity. Such programs have shown great promise as studies have revealed significant improvements in functional ability and quality of life and a reduction in healthcare costs and mortality in patients engaged in exercise programs $[2,4]$. Consequently, current recommendations on the management of CHF patients strongly emphasize an active lifestyle, and if possible, participation in a moderate planned physical activity regimen [5-7]. Despite the benefits of physical activity for CHF patients, research indicates few individuals with this condition actually engage in regular physical activity $[8,9]$. Numerous studies have revealed that traditional methods of promoting physical activity have been only marginally successful in CHF patients, citing failure to adhere to recommendations as a predominant factor $[10,11]$.

Literature suggests it is unrealistic to expect patients to make changes they are not prepared to make and little attention has focused on assessing the motivation and readiness of CHF patients to make necessary behavioral changes [8]. Application of behavioral theories, such as the Transtheoretical model (TTM) of behavior change 
may be useful in designing effective intervention programs for patients with heart failure $[6,12,13]$. These approaches could be used to guide strategies that go beyond traditional patient educational approaches [14]. For example, with the appropriate activities to meet the patient's stage of readiness for change, healthcare providers may facilitate a patient's movement along the continuum of change to alter lifestyle behaviors, such as physical activity, that can result in improved outcomes $[13,15,16]$.

Importantly, patients' perceptions of his/her stage of readiness might not always match their actual behavior [8]. Therefore, assessment of daily physical activity or exercise tolerance should be included to compare results of such assessments with the individual's stage of readiness. One of the better predictors of a patient's functional ability, exercise tolerance and physical activity behavior is the maximum distance an individual can walk on a 6-min walk test [17-21]. The 6-min walk test is a useful, simple, noninvasive alternative for assessing physical activity in CHF patients $[19,20]$ and provides prognostic information $[19,21,22]$ about the patient and is sensitive to interventions such as exercise training. There currently are no studies that have examined the TTM constructs for physical activity behavior to an actual measure of daily activity and exercise tolerance in CHF patients. Such data could potentially provide a better understanding of the links between the TTM constructs and the patient's physical activity behavior.

The specific aims of this study were: 1) To examine stage of readiness for planned physical activity; 2) To compare estimated self-reported daily physical activity and exercise tolerance/capacity by stage of readiness and 3) To determine the association between the stages of readiness for planned physical activity, self-reported daily physical activity and exercise tolerance, in patients with CHF. It was hypothesized the majority of patients would be in the pre-action stage of readiness; and that daily physical activity and six minute walking distance would be higher in those classified in the action and maintenance stages of readiness for planned physical activity.

\section{METHODS}

\subsection{Participants}

Patients with a diagnosis of heart failure were recruited from the Baton Rouge, LA and Jackson, MS areas. Participants had to be at least 18 years of age, and be on stable and optimal pharmacotherapy for their heart failure condition as determined by their physician. In addition, participants had to have the ability to read and write English. Participants were not excluded on the basis of gender, race, and/or etiology of heart failure. Exclusion criteria included individuals with acute medical conditions related or independent of the primary diagnosis (e.g. congestive heart failure requiring hospitalization, unstable angina, active infections etc.). Prior to participation, each individual was required to complete an informed consent approved by the area Institutional Review Board.

\subsection{Study Design}

The study was a cross-sectional design aimed the stage of change for physical activity in individuals with $\mathrm{CHF}$. Upon arrival to a scheduled Cardiology appointment, each participant was asked at check-in if they wanted to participate. If willing, they were given the informed consent approved by the area Institutional Review Board. Subsequently, the individual was given a folder containing all questionnaires to be completed for the study, including stage of change, and the Daily Activity in Heart Failure Questionnaire (DAIHFQ) [23,24]. Additionally, each participant underwent a six minute walk (6MWD) test and a review of medical history and demographics.

\subsection{Experimental Methods}

Stage of Change: Each participant's stage of change for physical activity adoption was assessed by the Stages of Change for Physical Activity Questionnaire, using the algorithm recommended by Reed and colleagues [25]. Specifically, participants were asked to indicate their present levels of physical activity behavior. Regular physical activity or exercise was defined as "any planned physical activity of moderate intensity (e.g., brisk walking, cycling, jogging, swimming, aerobics, etc.) aimed at improving/maintaining your health. The activity does not have to be painful to be effective but should be done at a level that increases your breathing rate and causes you to break a sweat. For activity to be regular it must add up to a total of 30 or more minutes per day, and be done at least 5 days per week. For example, you could take a 30 minute walk or take 3 ten minute walks each day." Participants were asked to indicate whether they engage in planned regular physical activity, according to the above definition, by marking one out of five statements, each of which reflects one of the five stages: precontemplation: "I do NOT plan to start regular physical activity in the next 6 months "; contemplation (C): "I am planning to start regular physical activity in the next six months"; preparation $(\mathrm{P})$ : Not regularly, but I engage in such activities occasionally and plan to start on a regular basis within the next month; action (A): "I have been physically active for LESS than six months"; and, maintenance (M): I have been physically active for MORE than six months". This staging measure is recommended as the most accurate estimate for adults [25,26], and the construct validity and test-retest reliability of this meth- 
odology has received strong support in previous research [26].

Physical Activity Questionnaire: Garet et al. published a detailed self-administered questionnaire of daily energy expenditure dealing with 7 dimensions of everyday life to better reflect habitual activities in patients with heart failure $[23,24]$. To validate this questionnaire the investigators $[23,24]$ measured actual $\mathrm{VO}_{2}$ peaks and found it to be a valid and reliable reflection of physical activity status. The original questionnaire was in French; therefore, in the present study a translation of the French questionnaire was used. The translation was made with help of the original investigators of the questionnaire with some modifications for appropriate physical activity choices for the US population. The questionnaire considers seven main areas including sleeping and resting, basic everyday activities (e.g. eating and washing), housework activities, leisure time physical activities, physical activity in salaried or voluntary work, moving about, and miscellaneous activities. Subsequently, activities could be classified in terms of intensity, i.e. activities below 3 METs, between 3 and 5 METs, and above 5 METs, as well as duration (minutes performed) [23]. Three response models were applied depending on the item. For example, participants reported information pertaining to time spent on each activity, quantifying the number of times the activity is done per week or day, and reported whether help or interruption was needed systematically, sometimes, or never during an activity [23]. Detailed scoring instructions are provided elsewhere [24].

Six-Minute Walk Test: Exercise intolerance is defined as the reduced ability to perform activities that involve dynamic movement of large skeletal muscles because of dyspnea or fatigue [17]. Exercise tolerance was measured as the maximum walking distance achieved on a 6-min-walk test [17]. The six-minute walk test is recommended as a useful, simple, noninvasive alternative for assessing physical activity in patients with heart failure. The test was performed in a corridor and participants were instructed to walk as far as possible in 6 minutes, avoiding chest pain, marked dyspnea, or other symptoms [27]. To standardize the protocol, the participants were not coached during the test, but made aware of time remaining to completion. The use of the six-minute walk test has received a lot of attention recently, due to its relative ease of administration. The test is also thought to reflect a realistic effort as performed in daily life, and appears highly acceptable to patients. Furthermore, several studies have reported the prognostic significance of the 6-minute walk test in patients with heart failure [18,20].

Estimated Exercise Capacity: Estimated $\mathrm{VO}_{2}$ peak was calculated from the 6-minute walking distance, us- ing a regression equation [21]. The equation: $\mathrm{VO}_{2}$ peak $=$ $0.03 \times 6$-minute walking distance (meters) $+3.98\left(\mathrm{r}^{2}=\right.$ 0.42 vs. actual $\mathrm{VO}_{2}$ peak measures) has been validated against actual $\mathrm{VO}_{2}$ peak measures and is based on the fact that the 6-minute walking distance is a significant predictor of $\mathrm{VO}_{2}$ peak [21].

\subsection{Statistical Analysis}

The Statistical Package for the Social Sciences (IBM, SPSS Statistics 20, Armonk, NY) was used for all data analyses. Descriptive statistics were performed to provide demographic characteristics of the study participants. To compare self-reported daily physical activity and exercise tolerance/capacity by stage of readiness a univariate general linear model was used. In addition, to determine the association between the stage of readiness for planned physical activity, self-reported daily physical activity and exercise tolerance simple correlation coefficients and a multivariate general linear model were used.

\section{RESULTS}

\subsection{Patient Characteristics}

Patient characteristics are presented in Tables 1 and 2. A total of 111 patients were studied. 81 participants were men, and 30 women. The mean age for the total group was $53 \pm 14$ (years), the average left ventricular ejection fraction (LVEF) for the group was $30.12 \pm 10.72 \%$. Etiology of heart failure included: ischemic heart disease (19\%), non-ischemic cardiomyopathy $(8 \%)$, hypertensive heart disease $(19 \%)$, viral induced heart failure $(3 \%)$, idiopathic cardiomyopathies (43\%), and congenital heart failure $(8 \%)$. The duration of heart failure averaged nearly 7 years, ranging from 6 months to 35 years. All patients were classified as New York Heart Association (NYHA) Class II (60\%) and III (40\%). Thirty-five percent of the patients were married, $35 \%$ were sepa$\mathrm{rated} /$ divorced or widowed, and the remaining $30 \%$ were single or engaged. Sixty-six percent of the participants were Caucasian, 32\% were African-American, and 2\% Hispanic.

\subsection{Stage of Change Classification}

The majority of participants reported being in the preparation stage of change $(n=41,27.7 \%)$, followed by contemplation $(\mathrm{n}=33,22.3 \%)$, maintenance $(\mathrm{n}=29$, $19.6 \%)$, action $(\mathrm{n}=23,15.5 \%)$, and precontemplation ( $\mathrm{n}$ $=22,14.9 \%$ ). In regards to the stage of change classification, no significant differences were noted for age, LVEF and heart failure duration. Furthermore, no differences in the stage classifications for race, employment status, income level, education level, heart failure diag- 
Table 1. Descriptive statistics (age, height, weight, BMI).

\begin{tabular}{lccccccccc}
\hline \multirow{2}{*}{ Stage of Change } & \multicolumn{2}{c}{ Age $(\mathrm{yrs})$} & \multicolumn{2}{c}{ Height $(\mathrm{m})$} & \multicolumn{2}{c}{ Weight $(\mathrm{kg})$} & \multicolumn{2}{c}{ BMI $\left(\mathrm{kg} / \mathrm{m}^{2}\right)$} \\
\cline { 2 - 9 } & Mean & SD & Mean & SD & Mean & SD & Mean & SD \\
\hline Precontemplation & 53 & 12 & 1.74 & 0.10 & 96.10 & 40.66 & 31.30 & 10.87 \\
Contemplation & 54 & 17 & 1.72 & 0.08 & 87.39 & 28.08 & 29.18 & 7.42 \\
Preparation & 53 & 12 & 1.75 & 0.10 & 105.29 & 31.82 & 34.11 & 10.10 \\
Action & 56 & 9 & 1.76 & 0.07 & 93.69 & 14.15 & 30.20 & 4.91 \\
Maintenance & 50 & 16 & 1.76 & 0.09 & 91.84 & 13.98 & 29.91 & 5.14 \\
Total & 53 & 14 & 1.74 & 0.09 & 94.31 & 26.36 & 30.82 & 7.73 \\
\hline
\end{tabular}

Table 2. Descriptive statistics (hemodynamics).

\begin{tabular}{ccccccccccc}
\hline \multirow{2}{*}{ Stage of Change } & \multicolumn{2}{c}{ LVEF $(\%)$} & \multicolumn{2}{c}{ HR (bts/min) } & \multicolumn{2}{c}{ SBP $(\mathrm{mmHg})$} & \multicolumn{2}{c}{ DBP $(\mathrm{mmHg})$} \\
\cline { 2 - 9 } & Mean & SD & Mean & SD & Mean & SD & Mean & SD \\
\hline Precontemplation & 33.38 & 13.06 & 73 & 13 & 130 & 22.91 & 73 & 14.93 \\
Contemplation & 29.55 & 12.79 & 75 & 20 & 115 & 26.99 & 71 & 18.99 \\
Preparation & 27.29 & 8.99 & 77 & 14 & 117 & 18.50 & 68 & 10.17 \\
Action & 31.37 & 7.55 & 72 & 12 & 110 & 15.90 & 70 & 6.85 \\
Maintenance & 31.35 & 11.22 & 75 & 11 & 121 & 8.51 & 69 & 6.69 \\
Total & 30.12 & 10.72 & 75 & 13 & 118 & 19.59 & 70 & 12.09 \\
\hline
\end{tabular}

nosis, number of co-morbidities and medications were observed. Therefore, these demographic variables were not treated as moderators in subsequent analyses.

\subsection{Daily Physical Activity and Stage of Readiness}

Table 3 presents the estimated minutes of rest and various intensities of physical activity per day for the heart failure patients per stage of readiness. The means and standard deviations for minutes spent in the different categories of activity indicate the majority of time individuals performed light activity ( 1 - 3 METs: $855 \pm 269$ $\mathrm{min} /$ day) followed by moderate activity (3 - 5 METs: $128 \pm 99$ minutes), and higher activity ( $>5$ METs: $15 \pm$ $19 \mathrm{~min} /$ day).

On the basis of a multivariate general linear model with the categories of activity as the dependent measures and stages of change as the fixed factor, a significant main effect was found $(p=0.0005)$. No differences were noted between the stage of change classification for rest and those activities considered between 1 - 3 METs. In contrast, patients classified in the Action and Maintenance stages of readiness performed significant more minutes in physical activities above 3 METs $(p=0.003)$ compared to those in the precontemplation/contemplation/preparation stages of readiness (see Figure 1).

\subsection{Six Minute Walk Distance and Stage of Readiness}

As indicated in Table 4, the average walking distance for the entire group was $349 \pm 118$ meters. The walking distance appears to increase dependent on the stage of change category. Indeed, the univariate general linear model analyses with the 6 minute walk distance as the dependent measure and stages of change as the fixed factor, revealed a significant main effect $(p=0.0001)$. Post hoc examination, using a Tukey test, indicates the following differences: The 6-minute walk distance for individuals in precontemplation, contemplation and preparation stages appear to be similar, whereas the 6-minute walk distance for the action and maintenance groups are significantly higher. Finally, there does not appear to be a significant difference between the action and maintenance groups. These findings suggest the 6-minute walk distance is capable of distinguishing the action and maintenance stages from the previous stages of readiness and is in support of the stated hypothesis.

\subsection{Estimated Exercise Capacity and Stage of Readiness}

Means and standard deviations for the estimated $\mathrm{VO}_{2}$ peak are presented in Table 4. Consistent with the 


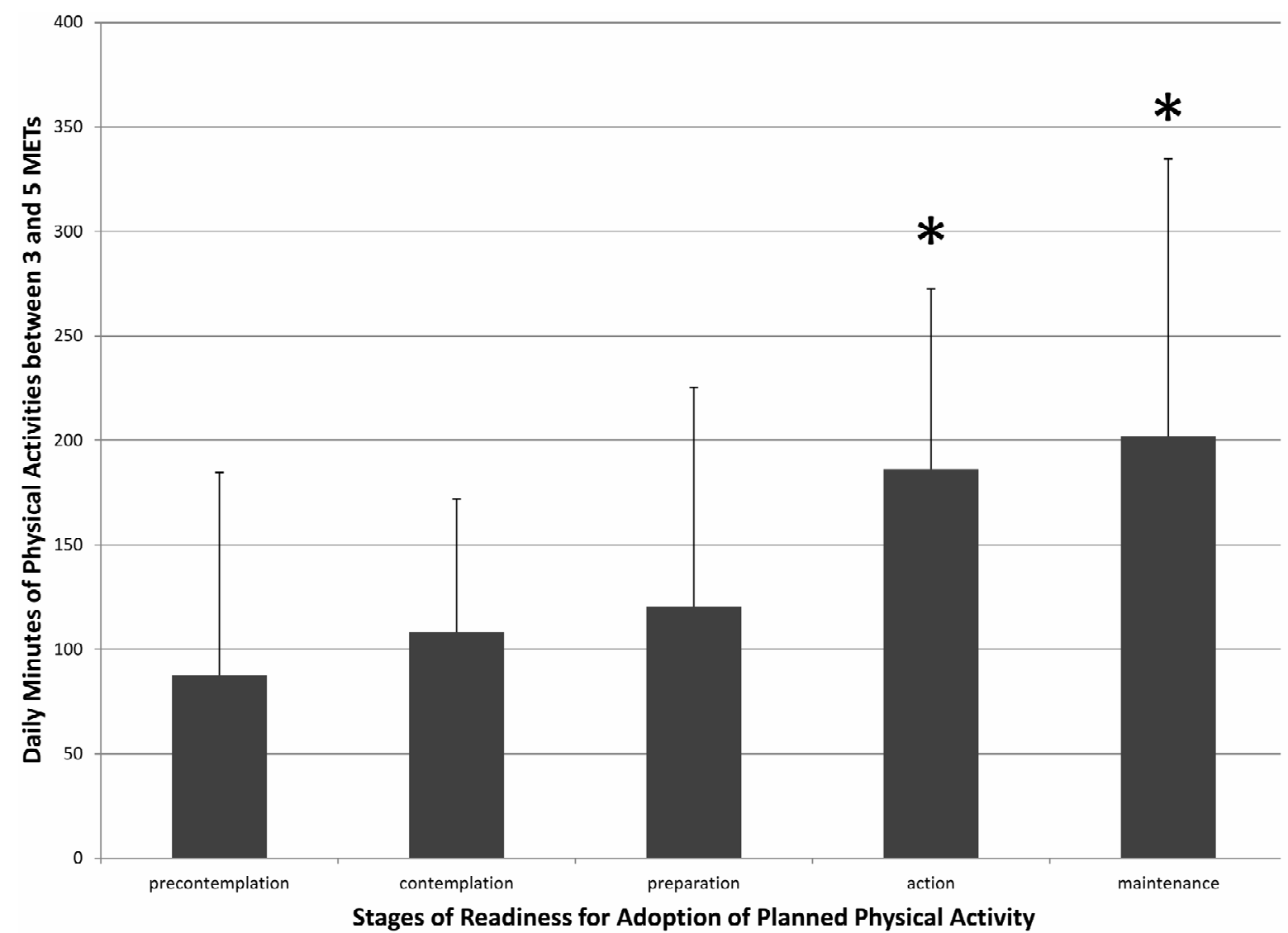

Figure 1. Estimated $\mathrm{VO}_{2}$ peak per stage of readiness. ${ }^{*} \mathrm{p}<0.05$ vs. precontemplation, contemplation, and preparation.

analysis for the 6-minute walk distance there was a significant main effect for stage of change category $(\mathrm{p}=$ $0.0004)$, and significant differences in the means for estimated $\mathrm{VO}_{2}$ peak between the Action and Maintenance categories vs. the remaining three categories. Importantly, the average estimated $\mathrm{VO}_{2}$ peak for the Action and Maintenance groups is above the prognostic relevant 14 $\mathrm{ml} / \mathrm{kg} / \mathrm{min}$ threshold (as indicated by the dashed line on Figure 2).

\subsection{Association between Six Minute Walk Test and Daily Physical Activity}

Simple correlation coefficients revealed significant associations between the 6-minute walk test and selfreported amount of minutes spent at rest $(\mathrm{r}=-0.27, \mathrm{p}=$ $0.02)$, performing activities between 3 and 5 METs $(r=$ $0.52, \mathrm{p}=0.0009)$, and activities over 5 METs $(\mathrm{r}=0.56, \mathrm{p}$ $=0.0001)$. A significant association was also seen between total minutes of daily physical activity and the 6 -minute walk test $(\mathrm{r}=0.32, \mathrm{p}=0.005)$.

\section{DISCUSSION}

\subsection{Patients Characteristics}

Review of the available literature reveals very few studies have attempted to examine the readiness for behavioral changes in CHF patients $[8,16]$. Consequently, it is difficult to compare the patient characteristics in the present study to other studies. Perhaps the only study of comparison is by Sneed and Paul [14]. In this study a mail survey was sent to patients with heart failure [14]. Respondents were asked to select the stage of readiness for change in six lifestyle behaviors important in management of the heart failure syndrome. Unfortunately, Sneed and Paul [8] did not examine the full TTM model among individuals with heart failure. Despite that, it is interesting to note the patient population in that study was remarkably similar to the present study [8].

Considering the present study also examined the association of the stage of change construct for physical activity with self-reported daily physical activity, and an actual measures of exercise tolerance (six minute walk test), a second comparison study is offered by Garet et al. [23]. These investigators assessed the reproducibility, sensitivity, and concurrent validity of self-reported daily energy expenditure in a population of stable CHF patients. Their population consisted of 105 Caucasian participants (Age: $55.8 \pm 12.4 \mathrm{yr}$, range 31 - 80 years). Mean LVEF was nearly exactly as reported in the present study (LVEF: $30.12 \% \pm 10.72 \%$ ). 
Table 3. Minutes of rest and various intensities of physical activity per day.

\begin{tabular}{|c|c|c|c|c|}
\hline Variable & Stage of Change & Mean & Std. Deviation & $\mathrm{n}$ \\
\hline \multirow{6}{*}{ Rest } & Precontemplation & 613 & 108 & 14 \\
\hline & Contemplation & 604 & 111 & 23 \\
\hline & Preparation & 531 & 137 & 24 \\
\hline & Action & 573 & 126 & 15 \\
\hline & Maintenance & 551 & 192 & 15 \\
\hline & Total & 572 & 137 & 91 \\
\hline \multirow{6}{*}{ Total Activity (min) } & Precontemplation & 820 & 224 & 14 \\
\hline & Contemplation & 995 & 223 & 23 \\
\hline & Preparation & $1030^{\dagger}$ & 339 & 24 \\
\hline & Action & $1133^{\dagger}$ & 341 & 15 \\
\hline & Maintenance & $1041^{\dagger}$ & 393 & 15 \\
\hline & Total & 1008 & 315 & 91 \\
\hline \multirow{6}{*}{ Minutes up to 3 METs } & Precontemplation & 684 & 230 & 14 \\
\hline & Contemplation & 879 & 201 & 23 \\
\hline & Preparation & 907 & 277 & 24 \\
\hline & Action & 927 & 312 & 15 \\
\hline & Maintenance & 824 & 299 & 15 \\
\hline & Total & 855 & 269 & 91 \\
\hline \multirow{6}{*}{ Minutes between 3 to 5 METs } & Precontemplation & 73 & 86 & 14 \\
\hline & Contemplation & 107 & 61 & 23 \\
\hline & Preparation & 111 & 101 & 24 \\
\hline & Action & $186^{*}$ & 86 & 15 \\
\hline & Maintenance & $180^{*}$ & 121 & 15 \\
\hline & Total & 128 & 99 & 91 \\
\hline \multirow{6}{*}{ Minutes above 5 METs } & Precontemplation & 2 & 7 & 14 \\
\hline & Contemplation & 8 & 13 & 23 \\
\hline & Preparation & 13 & 14 & 24 \\
\hline & Action & $20^{*}$ & 14 & 15 \\
\hline & Maintenance & $37^{*}$ & 27 & 15 \\
\hline & Total & 15 & 19 & 91 \\
\hline
\end{tabular}

${ }^{*} \mathrm{p}<0.05$ compared to precontemplation/contemplation/preparation; ${ }^{\dagger} \mathrm{p}<0.1$ compared to precontemplation/contemplation.

Table 4. Descriptive statistics: exercise tolerance (six minute walking distance (meters)) and estimated $\mathrm{VO}_{2}$ peak.

\begin{tabular}{lccccc}
\hline \multirow{2}{*}{ Stage of Change } & \multicolumn{2}{c}{6 MWD } & \multicolumn{4}{c}{$\begin{array}{c}\text { Estimated } \mathrm{VO}_{2} \\
\text { peak }\end{array}$} & $\mathrm{n}$ \\
\cline { 2 - 6 } & Mean & SD & Mean & SD \\
\hline Precontemplation & 297 & 137 & 12.90 & 4.11 & 12 \\
Contemplation & 276 & 108 & 12.24 & 3.22 & 29 \\
Preparation & 323 & 80 & 13.66 & 2.40 & 30 \\
Action & $401^{*}$ & 87 & $16.01^{*}$ & 2.60 & 20 \\
Maintenance & $472^{*}$ & 73 & $18.15^{*}$ & 2.20 & 20 \\
Total & 349 & 118 & 14.44 & 3.54 & 111 \\
\hline
\end{tabular}

${ }^{*} \mathrm{p}<0.05$ vs. precontemplation, contemplation, and preparation.

In summary, the present population characteristics appear to be similar to published studies, and based on the available guidelines for the management of heart failure, it would appear that the participants in this study would be excellent candidates for heart failure intervention programs including exercise training [6,9].

\subsection{Stage of Readiness}

Classification according to the TTM for stage of change reveals the majority of participants in this study were in the preparation stage of change $(\mathrm{n}=41,27.7 \%)$, followed by contemplation ( $\mathrm{n}=33,22.3 \%)$, maintenance $(\mathrm{n}=29,19.6 \%)$, action $(\mathrm{n}=23,15.5 \%)$, and precontemplation $(\mathrm{n}=22,14.9 \%)$. These findings are somewhat similar to a previous study which examined the predictors of exercise adherence and the validity of the Stages 
model among older adults $(\mathrm{n}=349)$ with a cardiac diagnosis (not heart failure) after discharge from a cardiac rehabilitation inpatient program [16]. In that study, 16\% of the patients were in precontemplation, $16 \%$ in contemplation, $22 \%$ in preparation, and $23 \%$ in both the action and maintenance stage [16]. In yet another study, the theorized associations of the TTM of behavior change constructs were examined along with exercise barriers, by stage of change for exercise behavior among individuals with physical disabilities [28]. In that study the majority of participants reported being in the maintenance stage of change $(53.7 \%)$, followed by precontemplation $(18.9 \%)$, action (11.8\%), contemplation $(9.0 \%)$, and preparation (6.5\%) [28]. One possible explanation for the greater number of individuals classified in the maintenance stage in the comparison study [28] is the passive recruitment method used by those authors, which tends to over-represent individuals with an interest in the subject matter. Other than differences in recruiting strategies, it is difficult to reconcile the differences among studies, given the vast differences in terms of populations, geographical location, and overall subject number. However, it is important to appreciate that in the current study $65 \%$ of patients were in the pre-action stages of readiness to engage in a treatment strategy known to be critical in the management of their disease. In fact, the present data fall in line with the study by Sneed \& Paul [8] who reported only $38 \%$ of patients with heart failure exercised regularly.

\subsection{Daily Physical Activity and Stage of Readiness}

Perhaps not surprising the amount of time spent in activities $>5$ METs in these patients is very low. This is not dissimilar from previous work from the Louisiana Healthy Aging Study [29]. In that study, a strong agerelated decline in levels of moderate intensity physical activity, and an increase in time spent in light intensity activity with increasing age, suggests higher intensity activities are being avoided or replaced by lower intensity activities. It is important to consider that decreased physical activity, in the elderly, is responsible for $46 \%$ of the decrease in total energy expenditure [30], and that a decline in daily physical activity predicts frailty and healthrelated disability [31].

The reason(s) for the low levels of moderate and higher intensity physical activity among the patients cannot be discerned from the present data but most likely include psychological and physiological factors. Previous studies report in independently living older adults a lack of interest in physical activity participation, and joint pain as possible deterrents [32]. Given the cardinal symptoms of CHF patients include chronic fatigue, shortness of breath and exercise intolerance it is understandable higher MET activities are to a large extent avoided.

Uniquely, these data suggest that individuals in the action and maintenance stages perform a greater amount of physical activity, especially at higher intensities, compared to those in the early stages of readiness (precontemplation, contemplation). Perhaps this indeed provides validation of the TTM constructs for physical activity for the CHF patients, or recognition of those patients to include physical activity in the management of their condition.

\subsection{Six Minute Walk Distance and Stage of Readiness}

A unique contribution of these data are the findings that the 6-minute walking distance is capable of distinguishing individuals in the action and maintenance stages from the early stages of readiness (precontemplation, contemplation). The 6-minute walk test carries important prognostic information. The total group average for the maximum walking distance (349 \pm 118 meters) in this study appears to be quite typical for patients with heart failure, with a NYHA Class II and III. For example, Opasich et al. reported a maximum walking distance of $396 \pm 92$ meters in 315 chronic heart failure patients (age: $53 \pm 9$ years, NYHA class: II (182), III (133)) [22].

Perhaps more importantly, based on the literature indicating that a maximum walking distance less than 300 meters is associated with an increased likelihood of early mortality $[19,20,22]$; the results of the present study suggest many of the patients are at elevated risk for complications [33]. In fact, $36 \%$ of all the participants in this study scored below the 300 meter threshold. Interestingly, patients who scored below $300 \mathrm{~m}$ on the 6-minute walk test were not different from the patients scoring above $300 \mathrm{~m}$, in terms of age, BMI, LVEF, resting blood pressure and heart rate. In addition, no differences were noted for race, employment status and income, etiology, and length of heart failure, co morbidities, or number of medications. One interesting observation in the present study is that a greater percentage of women scored below the 300 meter threshold. Given the evidence that the distance of $<300 \mathrm{~m}$ also identifies women at high risk of death [19], this requires further examination and focus from a clinical perspective. A further important observation is that the average age for the women in this study was significantly lower than the men, yet their average maximum walking distance was much lower as well.

In regards to the stated hypothesis, it was predicted that the maximum walking distance would distinguish the action and maintenance stages from the precontemplation and contemplation stages of readiness. Indeed the 
action and maintenance stages were different from the precontemplation and contemplation stages of readiness. Closer observation of these data reveal that the majority of patients in the action and maintenance stages were in fact above the 300meter, compared to the majority of patients in the early stages (i.e., precontemplation, contemplation) who were below this clinically important cut-off. More specifically, $51 \%$ of patients in the precontemplation stages were below the 300 meter, compared to only $7.5 \%$ in the action/maintenance stages. These data suggest many of CHF patients who are in the pre-action stages of readiness for physical activity are at elevated risk for complications.

\subsection{Estimated Exercise Capacity and Stage of Readiness}

Using the 6-minute walking distance to estimate $\mathrm{VO}_{2}$ peak reveals that a greater majority of those individuals above $14 \mathrm{ml} / \mathrm{kg} / \mathrm{min}$ are in the action and maintenance categories (see Figure 2). More specifically, $71 \%$ of patients in the precontemplation stages were below the $14 \mathrm{ml} / \mathrm{kg} / \mathrm{min}$ threshold compared to only $7.5 \%$ in the Action/Maintenance stages. Again these data sug- gest that the majority of patients with heart failure in the early stages of readiness are below the $14 \mathrm{ml} / \mathrm{kg} / \mathrm{min}$ threshold, a powerful prognostic indicator of an increased likelihood of death or hospitalization within 3 months to a year $[21,33]$.

\subsection{Association between Six Minute Walk Test and Daily Physical Activity}

Recognizing that the questionnaire was translated and slightly modified, the data did allow further comparison to the functional scores. The findings revealed significant associations between the 6-minute walk test and the self-reported amount of minutes spent at rest $(\mathrm{r}=-0.27$, $\mathrm{p}=0.02)$, performing activities between 3 and 5 METs $(r$ $=0.52, \mathrm{p}=0.0009)$, activities over 5 METs $(\mathrm{r}=0.56, \mathrm{p}=$ $0.0001)$. Moreover, a significant association was apparent between the total minutes of physical activity performed throughout the day, and the 6-minute walk test ( $\mathrm{r}$ $=0.32, \mathrm{p}=0.005)$. This is the first study to report these associations using a submaximal performance test. In comparison to previous work [23] the reported associations are somewhat lower. The discrepancy may in part be a consequence of the questionnaire modifications, or

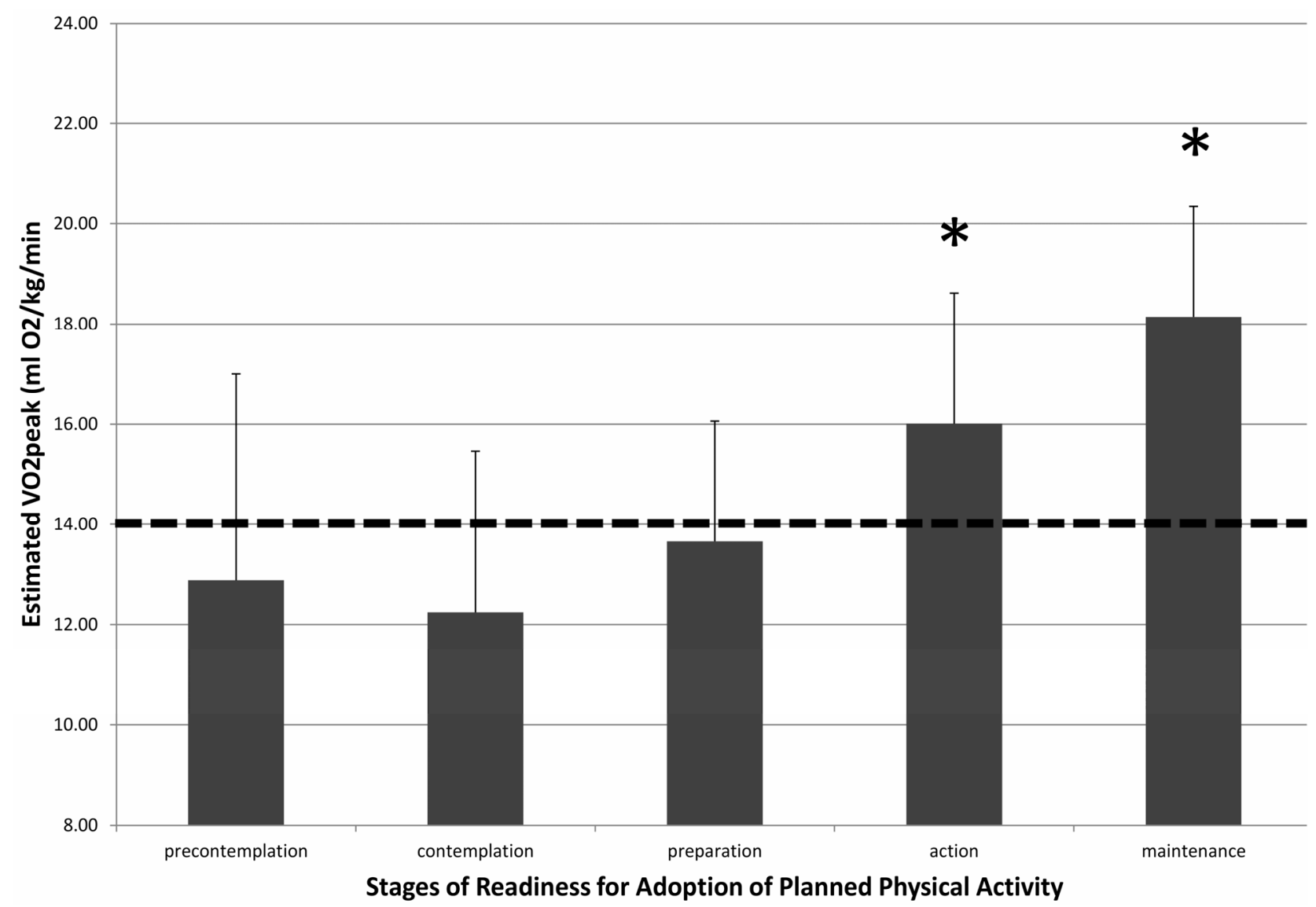

Figure 2. Estimated minutes of physical activity between 3 and 5 METs according to stage of readiness. ${ }^{*}$ p $<0.05$ vs. precontemplation, contemplation, and preparation. 
the fact that the 6-minute walk test was used. Irrespective of those possibilities, the findings are intriguing and suggest the questionnaire is able to differentiate between patients who score high or low on the 6-minute walk test. Interestingly, the patients in the present study had lower estimated $\mathrm{VO}_{2}$ peak values, than their French counterparts. In addition, the patients in this study performed fewer activities above 5 METs and had slightly lower overall energy expenditures over the course of the day. It is difficult to reconcile these differences as the US patients were similar in terms of age, gender, LVEF, NYHA class, and etiology of disease. However, there are apparent differences in terms of weight and BMI, with the American cohort heavier (American Cohort: $95.28 \pm$ 26.43; French Cohort: $74.80 \pm 15.00)$ and with a greater average BMI (American Cohort: $31.68 \pm 8.53$; French Cohort: $25.08 \pm 4.00$ ).

These data suggest that those in the early stages are clinically more fragile, are less involved in daily physical activities and are women. The importance of this information obviously lies in the fact that specific strategies need to be developed to move patients in these pre-action stages toward the Action stage.

In conclusion, data from this study indicate that the majority of CHF patients are in the pre-action stages of readiness for adoption of planned physical activity. In addition, CHF patients in pre-action stages of readiness are engaged in less self-reported daily activity compared and have significant lower exercise tolerance/capacity than those in the action and maintenance stages. The fact that a significantly higher number of CHF patients in preaction stages scored $<300$ meters on the six-minute walk test and have an estimated $\mathrm{VO}_{2}$ peak of $<14 \mathrm{ml} / \mathrm{kg} / \mathrm{min}$, indicates these patients are clinically more fragile and at greater risk for complications and early mortality. These data suggest greater clinical emphasis should be placed on strategies to move patients toward the action and maintenance stages of readiness.

\section{REFERENCES}

[1] McAlister, F.A., Lawson, F.M., Teo, K.K. and Armstrong, P.W. (2001) Systematic review of randomized trials of disease management programs in heart failure. American Journal of Medicine, 110, 378-384. doi:10.1016/S0002-9343(00)00743-9

[2] Rich, M.W. (1999) Heart failure disease management: A critical review. Journal of Cardiac Failure, 5, 64-75. doi:10.1016/S1071-9164(99)90026-X

[3] Knox, D. and Mischke, L. (1999) Implementing a congestive heart failure disease management program to decrease length of stay and cost. Journal of Cardiovascular Nursing, 14, 55-74.

[4] Piepoli, M.F., Davos, C., Francis, D.P. and Coats, A.J. (2004) ExTraMATCH Collaborative. Exercise training meta-analysis of trials in patients with chronic heart failure (ExTraMATCH). British Medical Journal, 24, 189. 196.

[5] Hunt, S.A., Baker, D.W., Chin, M.H., Cinquegrani, M.P., Feldman, A.M., Francis, G.S., Ganiats, T.G., Goldstein, S., Gregoratos, G., Jessup, M.L., Noble, R.J., Packer, M., Silver, M.A. and Stevenson, L.W. (2001) ACC/AHA guidelines for the evaluation and management of chronic heart failure in the adult: Executive summary. Circulation, 104, 2996-3007. doi:10.1161/hc4901.102568

[6] Parish, T.R. and Welsch, M.A. (2007) Exercise training for the heart failure patient: Is your patient ready? Cardiopulmonary Physical Therapy Journal, 18, 12-20.

[7] Pina, I.L., Apstein, C.S., Balady, G.J., Belardinelli, R., Chaitman, B.R., Duscha, B.D., Fletcher, B.J., Fleg, J.L., Myers, J.N. and Sullivan, M.J. (2003) American heart association committee on exercise, rehabilitation, and prevention. Exercise and heart failure: A statement from the AHA committee on exercise, rehabilitation, and prevention. Circulation, 4, 1210-1225. doi:10.1161/01.CIR.0000055013.92097.40

[8] Sneed, N.V. and Paul, S. (2004) Strategies for behavior change in patients with heart failure. American Journal of Critical Care, 13, 305-313.

[9] Suppan, J. (2001) Using the transtheoretical approach to facilitate change in the heart failure population. Congestive Heart Failure, 7, 151-155. doi:10.1111/j.1527-5299.2001.00244.x

[10] Ghali, J.K., Kadakia, S., Cooper, R. and Ferlinz, J. (1998) Precipitating factors leading to decompensation of heart failure: Traits among urban blacks. Archives of Internal Medicine, 148, 2013-2016. doi:10.1001/archinte.1988.00380090087021

[11] Happ, M.B., Naylor, M.D. and Roe-Prior, P. (1997) Factors contributing to rehospitalization of elderly patients with heart failure. Journal of Cardiovascular Nursing, 11, 75-84.

[12] Konstam, M.A., Dracup, K., Baker, D.W., Bottorff, M.B., Brooks, N.H., Dacey, R.A., Dunbar, S.B., Jackson, A.B., Jessup, M. and Johnson, J.C. (1995). Heart failure: Evaluation and care of patients with left ventricular systolic dysfunction. Journal of Cardiac Failure, 1, 183-187. doi:10.1016/1071-9164(95)90021-7

[13] Spirduso, W.W. and Cronin, D.L. (2001) Exercise doseresponse effects on quality of life and independent living in older adults. Medicine and Science in Sports and Exercise, 33, S598-S608. doi:10.1097/00005768-200106001-00028

[14] Dunbar, S.B., Jacobson, L.H. and Deaton, C. (1998) Heart failure: Strategies to enhance patient self-management. AACN Clinical Issues, 9, 244-256. doi:10.1097/00044067-199805000-00007

[15] Rostagno, C., Olivo, G., Comeglio, M., Boddi, V., Banchelli, M., Galanti, G. and Gensini, G.F. (2003) Prognostic value of 6-minute walk corridor test in patients with mild to moderate heart failure: Comparison with other methods of functional evaluation. European Journal of Heart Failure, 5, 247-252. doi:10.1016/S1388-9842(02)00244-1 
[16] Hellman, E.A. (1997) Use of the stages of change in exercise adherence model among older adults with a cardiac diagnosis. Journal of Cardiopulmonary Rehabilitation, 7, 145-155. doi:10.1097/00008483-199705000-00001

[17] Guyatt, G.H., Sullivan, M.J., Thompson, P.J., Fallen, E.L., Pugsley, S.O., Taylor, D.W. and Berman, L.B. (1985) The 6-minute walk: A new measure of exercise capacity in patients with chronic heart failure. Canadian Medical Association Journal, 132, 919-923.

[18] Milligan, N.P., Havey, J. and Dossa, A. (1997) Using a 6-minute walk test to predict outcomes in patients with left ventricular dysfunction. Rehabilitative Nursing, 22, 177-181. doi:10.1002/j.2048-7940.1997.tb02095.x

[19] Rostagno, C., Olivo, G., Comeglio, M., Boddi, V., Banchelli, M., Galanti, G. and Gensini, G.F. (2003) Prognostic value of 6-minute walk corridor test in patients with mild to moderate heart failure: Comparison with other methods of functional evaluation. European Journal of Heart Failure, 5, 247-252. doi:10.1016/S1388-9842(02)00244-1

[20] Bittner, V., Weiner, D.H., Yusuf, S., Rogers, W.J., McIntyre, K.M., Bangdiwala, S.I., Kronenberg, M.W., Kostis, J.B., Kohn, R.M., Guillotte, M., et al. (1993) Prediction of mortality and morbidity with a 6-minute walk test in patients with left ventricular dysfunction. SOLVD Investigators. Journal of the American Medical Association, 13, 1702-1707. doi:10.1001/jama.1993.03510140062030

[21] Cahalin, L.P., Mathier, M.A., Semigran, M.J., Dec, G.W. and DiSalvo, T.G. (1996) The six-minute walk test predicts peak oxygen uptake and survival in patients with advanced heart failure. Chest, 110, 325-332. doi:10.1378/chest.110.2.325

[22] Opasich, C., Pinna, G.D., Mazza, A., Febo, O., Riccardi, R., Riccardi, P.G., Capomolla, S., Forni, G., Cobelli, F. and Tavazzi, L. (2001) Six-minute walking performance in patients with moderate-to-severe heart failure; is it a useful indicator in clinical practice? European Heart Journal, 22, 488-496. doi:10.1053/euhj.2000.2310

[23] Garet, M., Barthelemy, J.C., Degache, F., Costes, F., Da-Costa, A., Isaaz, K., Lacour, J.R. and Roche, F. (2004) A questionnaire-based assessment of daily physical activity in heart failure. European Journal of Heart Failure, 6, $577-584$.

[24] Garet, M., Degache, F., Costes, F., Da-Costa, A., Lacour, J., Barthelemy, J. and Roche, F. (2004) DAQIHF: Meth- odology and validation of a daily activity questionnaire in heart failure. Medicine and Science in Sports and Exercise, 36, 1275-1282. doi:10.1249/01.MSS.0000135776.09613.0D

[25] Reed, G.R., Velicer, W.F., Prochaska, J.O., Rossi, J.S. and Marcus, B.H. (1997) What makes a good staging algorithm: Examples from regular exercise. American Journal of Health Promotion, 12, 57-66. doi:10.4278/0890-1171-12.1.57

[26] Cardinal, B.J. (1997) Construct validity of stages of change for exercise behavior. American Journal of Health Promotion, 12, 68-74. doi:10.4278/0890-1171-12.1.68

[27] Rostagno, C., Galanti, G., Romano, M., Chiostri, G. and Gensini, G.F. (2002) Prognostic value of 6-minute walk corridor testing in women with mild to moderate heart failure. Italian Heart Journal, 3, 109-113.

[28] Cardinal, B.J., Kosma, M. and McCubbin, J.A. (2004) Factors influencing the exercise behavior of adults with physical disabilities. Medicine and Science in Sports and Exercise, 36, 868-875. doi:10.1249/01.MSS.0000126568.63402.22

[29] Johannsen, D.L., DeLany, J.P., Frisard, M.I., Welsch, M.A., Rowley, C.K., Fang, X., Jazwinski, S.M., Ravussin, E., for the Louisiana Healthy Aging Study (2008) Physical activity in aging: Comparison among young, aged, and nonagenarian individuals. Journal of Applied Physiology, 105, 495-501. doi:10.1152/japplphysiol.90450.2008

[30] Black, A.E., Coward, W.A., Cole, T.J. and Prentice, A.M. (1996) Human energy expenditure in affluent societies: An analysis of 574 doubly-labeled water measurements. European Journal of Clinical Nutrition, 50, 72-92.

[31] Sneed, N.V. and Paul, S.C. (2003) Readiness for behavior change in heart failure. American Journal of Critical Care, 12, 444-453.

[32] Crombie, I.K., Irvine, L., Williams, B., McGinnis, A.R., Slane, P.W., Alder, E.M. and McMurdo, M.E. (2004) Why older people do not participate in leisure time physical activity: A survey of activity levels, beliefs and deterrents. Age and Ageing, 33, 287-292. doi:10.1093/ageing/afh089

[33] Welsch, M.A. and Parish, T.R. (2004) The Heart Failure Syndrome: The role of exercise training. Cardiopulmonary Physical Therapy Journal, 5, 3-14. 